Doi: $10.32481 / \mathrm{djph} .2021 .09 .014$

\title{
Prevention of and Early Intervention for Cognitive Decline Due to Alzheimer's Disease and Related Disorders
}

\author{
Matthew L. Cohen, $\mathrm{PhD}^{1}$; Alexis C. Ryan, MA; ${ }^{2}$ Alyssa M. Lanzi, PhD, CCC-SLP ${ }^{3}$ \\ 1. Department of Communication Sciences \& Disorders, Delaware Center for Cognitive Aging \\ Research, Center for Health Assessment Research and Translation, University of Delaware
}

2. ChristianaCare

3. Delaware Center for Cognitive Aging Research, Center for Health Assessment Research and Translation, University of Delaware

An expected consequence of aging is the decline of so-called "fluid" cognitive abilities, including attention, processing speed, working memory, episodic memory, and executive functions. ${ }^{1,2}$ An average 85-year-old is not expected to perform as quickly or as accurately on tasks requiring these cognitive abilities as an average 25 -year-old. As a result, everyday cognitive errors and inefficiencies such as slow word retrieval or walking into a room and forgetting one's intention, which happen to everyone, ${ }^{3}$ become more frequent and significant with age. ${ }^{4,5}$ The biological and cognitive mechanisms of age-related cognitive decline are multifactorial and not entirely understood, but a growing body of evidence describes risk factors that can be mitigated and protective factors that can be enhanced to preserve cognition or slow the rate of decline. ${ }^{6}$ These same protective factors for brain health may be effective in mitigating the impact that Alzheimer's disease has on a person's daily functioning, potentially delaying their transition to dementia. ${ }^{7}$

Alzheimer's disease (AD) is not a normal consequence of aging, although it does affect $10 \%$ of adults over 65 , including $32 \%$ of those over 85 , making it the most common cause of mild cognitive impairment (MCI) and dementia in older adults. ${ }^{8} \mathrm{MCI}$ is a state at which a person has greater cognitive decline than expected for their age, but their cognitive weaknesses have not overpowered their ability to compensate for them. ${ }^{9}$ A person with dementia, on the other hand, is not able to successfully compensate for their cognitive weaknesses and therefore has functional challenges in everyday life and during completion of activities of daily living. Although symptom-alleviating drugs have been approved by the FDA, there are unfortunately no curative or disease-modifying pharmacological treatments for MCI or dementia from AD at this time.

Given the lack of definitive pharmacological treatments, research priorities are focused on preventing future cases through risk reduction, improving quality of life, improving the dementia workforce and quality of care, and optimizing the quality and inclusivity of health and social care systems. ${ }^{10}$ The importance of risk reduction, in particular, has been highlighted by recent evidence reporting that modifiable risk factors attribute to approximately $40 \%$ of dementia cases worldwide. ${ }^{7}$ One particularly new and noteworthy finding is the impact of untreated hearing loss, which was found to confer more individual risk for dementia than any other risk factor. ${ }^{7}$ Identifying atypically declining older adults early in the progression of the disease, creates an opportunity for the implementation of secondary prevention interventions that focus on delaying the transition to dementia (i.e., loss of independence). 


\section{Evidence for Multicomponent Interventions}

Behavioral preventive interventions focus on promoting changes in lifestyle that improve overall health. ${ }^{11}$ In general, evidence for behavioral interventions in isolation (e.g., physical activity, psychosocial support, or cognitive training) is mixed - some studies report modest improvements in cognition while others find no significant benefit. ${ }^{7}$ The mixed findings are most likely due to the heterogeneity of intervention details, outcome measures, and case definitions. But because of the mixed evidence for each component in isolation, interest has turned to the efficacy of multicomponent interventions for people at risk for atypical cognitive decline with the intent to delay or prevent conversion to dementia.

The Finnish Geriatric Intervention Study to Prevent Cognitive Impairment and Disability (FINGER) has provided the strongest evidence to date for the beneficial effect of a multicomponent behavioral intervention for adults at risk for AD. This multicomponent intervention consisted of nutritional guidance, exercise, cognitive rehabilitation, and intensive monitoring and management of metabolic and vascular risk factors (e.g., diabetes, hypertension, hyperlipidemia). ${ }^{12}$ The researchers conducted a double-blind randomized controlled trial of 1260 adults (aged 60-77) at elevated risk for dementia to examine the efficacy of their intervention. The main finding was that participants in the intervention condition improved more on a composite score of performance-based cognitive measures than those in a control group who received only health education, counseling, and monitoring. ${ }^{13}$ The encouraging findings from this clinical trial have placed the FINGER research team at the forefront of guiding international efforts for AD risk reduction. In fact, in 2020, the Worldwide FINGERS Network included investigators from 25 countries conducting trials to adapt, test, and optimize the FINGER framework for various cultural and economic settings. ${ }^{14}$ Furthermore, the strong findings from this trial have led to the initiation of numerous other clinical trials investigating multicomponent interventions, many of which are still enrolling participants. ${ }^{6}$

Two other large-scale clinical trials testing multicomponent interventions have been completed. The Dutch PreDIVA study cluster-randomized 3526 older adults (aged 70-79) to usual care or a 6 -year nurse-led cardiovascular intervention consisting of lifestyle advice. ${ }^{15}$ This study found no significant benefit of the intervention, but investigators speculated that the lack of dementia prevention may have reflected good baseline cardiovascular health and high standards of usual care. In another trial, the Multidomain Alzheimer Preventive Trial (MAPT), 1680 adults (age $70+$ ) with memory complaints were randomly assigned to one of four groups. ${ }^{16}$ One group received a multidomain lifestyle intervention consisting of 43 group sessions of cognitive training, physical activity, and nutrition, and three preventative consultations. A second group received only nutritional supplementation with omega- 3 fatty acids. A third group received both, and a fourth group received only a placebo capsule. The results were nuanced. Although there were not significant differences between any of the three intervention groups compared to placebo, post-hoc exploratory analyses revealed that when both groups receiving the multidomain intervention were pooled, they showed less cognitive decline than the other groups, particularly in participants with higher cardiovascular risk or amyloid positivity. ${ }^{17,18}$

The encouraging findings of the FINGER and MAPT studies compared with the null findings from the preDIVA study suggest the importance of active interventions. The passive health advice and monitoring provided to control group participants did not lead to benefits.

Unfortunately, several aspects of the FINGER, MAPT, and similar multicomponent intervention studies make them difficult to implement in real-world clinical practice settings. For example, 
the length of the FINGER study intervention was two years, during which participants were expected to attend more than 430 in-person sessions (155 in-person cognitive training sessions, 264 in-person physical training sessions, 10 in-person nutritional counseling sessions, and 10 inperson visits related to management of metabolic and vascular risk factor). This would be financially and logistically impossible for most community-dwelling older adults, especially those in underserved communities who are in fact at the highest risk for dementia. ${ }^{8,19-21}$ Indeed, even given all of the financial and logistical support received by FINGER study participants, treatment adherence was nonetheless very poor, especially for the cognitive training sessions: only $12 \%$ of participants completed all sessions, $20 \%$ of participants completed half, and $37 \%$ of participants did not attend a single training session. ${ }^{22}$ Similarly, $40 \%$ of participants could not or chose not to adhere to the standardized physical training component. ${ }^{23}$

Another major barrier to implementation of a multicomponent intervention in clinical settings is that these programs may not be well-suited to individuals' needs or capabilities. The intervention components are often structured, and lack person-centeredness, as participants are expected to adhere to each component. For example, in the FINGER study, all participants were expected to complete the 264 sessions of physical training, regardless of their baseline fitness levels, personal goals, and health risk factors. Acknowledging these limitations, the ongoing "Maintain Your Brain" study in Australia assigns participants only to those intervention modules that address that person's risk factors, making it somewhat more person-centered. ${ }^{24}$ This intervention is also unique because it is delivered entirely through a custom online platform (self-paced video library), making it less burdensome, more accessible to rural communities, and more scalable. Whether engagement/adherence will be helped by improved accessibility remains to be seen. In addition, passive rather than active delivery of the intervention may be less engaging for some participants.

Finally, most multicomponent intervention studies are limited by their focus on decontextualized, performance-based cognitive test scores as outcome measures and the use of restorative rather than compensatory cognitive training. Restorative cognitive interventions (or "cognitive training" interventions) are those that attempt to repair weak cognitive domains (e.g., working memory), often through performance of rote tasks. A 2019 Cochrane Review of restorative cognitive interventions for mild to moderate dementia analyzed 32 studies that were conducted between 1988 and 2018. ${ }^{25}$ The findings depended on the control condition. When cognitive training was compared with a no-intervention control group or condition, the authors found moderate-quality evidence for a small to moderate effect of cognitive training on composite measures of global cognition, and high-quality evidence of a moderate effect on semantic fluency tasks specifically that persisted for 3-12 months after treatment. However, due to very poor evidence quality, it was not possible to determine whether the cognitive training interventions were associated with any meaningful gains on clinical disease severity.

When cognitive training was compared with alternative treatments, the authors found little evidence for an effect of treatment on global cognitive functioning, but this was only based on low quality evidence. However, the authors reported being moderately confident that restorative cognitive interventions were not associated with improvement beyond the alternative treatments on outcomes related to mood, behavioral or psychology symptoms, or daily functioning.

There is more encouraging evidence for this form of cognitive training when it is initiated before significant declines have occurred. The Advanced Cognitive Training for Independent and Vital Elderly (ACTIVE) trial, which randomly assigned 2832 older adults (aged 65-94) to one of three 
restorative training interventions: memory, reasoning, or processing speed. The intervention consisted of ten group sessions plus four booster sessions. The main findings were that each intervention improved participants' completion of the trained cognitive task (for up to ten years in the case of speed and reasoning training), but with unclear effect on everyday living skills. At least $60 \%$ of participants, across every intervention group, reported less difficulty performing everyday living tasks compared to non-trained participants. However, those group differences were not observed on performance-based measures of daily function. ${ }^{26}$

Compensatory cognitive interventions, on the other hand, do not attempt to repair cognitive substrates, but rather teach the use of strategies and aids (e.g., calendars and note-taking systems) to directly improve adults' completion of everyday living tasks. Compensatory interventions have the advantage of being more person-centered than traditional cognitive training by allowing the individual to focus on specific everyday challenges that are meaningful to them in a way that leverages their cognitive strengths and existing practices. ${ }^{27,28}$ And because functional independence is the key difference between MCI and dementia, preserving one's independent living skills by definition delays the transition to dementia. ${ }^{29}$

The multicomponent Mayo Clinic Healthy Action to Benefit Independence and Thinking (HABIT) program is relatively unique because it includes both compensatory and restorative components and focuses on quality-of-life as the primary outcome rather than cognitive test scores. A comparative effectiveness trial of HABIT components recently evaluated the relative efficacies of different combinations of components on quality-of-life at one year. ${ }^{30}$ Results indicated that wellness education seemed to be the most effective of the components, and restorative cognitive therapy was the least. It is unfortunate that the preDIVA intervention (nurse-led lifestyle counseling) did not study quality-of-life outcomes other than depression symptoms (no effect of treatment), because it may have been more impactful on a person's overall health and well-being than fully appreciated. On the other hand, perhaps the wellness education component of the HABIT was uniquely effective for QoL because it was led by psychologists, focused on self-efficacy (a priority for people with $\mathrm{AD}^{31}$ ), and focused on wellness beyond strictly cardiovascular health. Interestingly, when asked directly about which component of the intervention was most important, persons with $\mathrm{MCI}$ (pwMCI) and their care partners ranked the compensatory cognitive intervention first - above all other components even though the wellness module was the most predictive of QOL at 1 year. ${ }^{31}$

\section{Early Identification and Intervention in the State of Delaware}

Although evidence for multicomponent interventions is still accumulating, there is a critical need and demand for services that can be implemented by clinicians working in healthcare settings with older adults at risk for and with early signs of dementia. The remaining sections of this article will provide a brief overview of some of the specific resources available to clinicians in the State of Delaware that focus on prevention and improving quality of life in older adults at risk for dementia. Readers are also directed to other articles in this issue.

\section{Memory Ambassadors - Brain Wellness Education and Memory Screening}

In 2017, a group called Memory Ambassadors was formed by clinicians within the Swank Center for Memory Care and Geriatric Consultation at ChristianaCare. This group travels to community sites (e.g., senior centers, senior living communities) to provide brain wellness education and free screenings of nutrition, balance, hearing, and memory to older adults 
throughout the State of Delaware. Faculty and clinicians who deliver this intervention are a collaborative, interdisciplinary team from ChristianaCare and the University of Delaware (UD), contributing skills from the fields of psychiatry, neuropsychology, speech-language pathology, social work, nutrition, and nursing.

The Memory Ambassador program begins with a series of interactive presentations from members of the team addressing brain wellness education, nutrition, and fall risk reduction. The cognitive aging presentation is organized around an acronym, D.A.N.C.E.R.S: Disease Management, Physical Activity, Nutrition, Cognitive Stimulation, Social Engagement, Relaxation, and Successful Sleep. As discussed elsewhere, ${ }^{32}$ Dr. Ellison created this acronym to help adults remember the evidence-based strategies for enhancing cognitive aging.

Following the presentations, the Memory Ambassadors' program offers free confidential memory screenings to interested attendees. The memory screenings, based on a self-report interview and a performance test, are conducted by a member of our team - and their supervised graduate students - and last approximately 10 minutes. At the end of the screening, the team member provides feedback about the attendee's performance and asks if they would like that information to be shared with their primary care clinician. Since 2017, close to 600 older adults have been screened by the program. In 2020, in the context of the COVID-19 pandemic, Memory Ambassadors transitioned to an online platform with good success. Interested older adults can sign up to attend an event free of charge, however, participation in online events requires stable internet and an electronic device. For more information on the Memory Ambassadors program, the reader is referred to the website: https://christianacare.org/es/swankmemory-ambassadors-program/.

Although the program began primarily as a service to the community, more systematic program evaluation is under way. This is important because the value of brain health education may be obscured by the use of education as a control condition in multicomponent intervention studies. For example, the control group in the FINGER trial met with the study nurse in person three times over 24 months, during which time the nurse took blood pressure, weight, hip and waist circumference, and blood samples. Participants in the control condition were then provided written information about the significance of these health metrics alongside advice for seeking medical care if needed. ${ }^{12}$ Interestingly, the findings from the FINGER trial indicated that participants in the control condition also improved their composite test score over 2 years, despite expected declines in cognition due to age. ${ }^{13}$ Of note, some have hypothesized that the small differences between the conditions may be due to the high quality of the wellness education for the control group - better than what is received by most older adults in the United States, especially given that many older adults do not have a primary care doctor or attend wellness exams. ${ }^{33,34}$ The value of brain health education is also indicated by the finding that across multicomponent interventions, the highest adherence rates have been observed for the components related to cardiovascular monitoring and nutritional counseling, ${ }^{23}$ suggesting these components are perceived to be useful and not burdensome. And the Mayo Clinic HABIT trial identified wellness education as the strongest predictor of Quality of Life at 1 year. ${ }^{30}$

\section{Proposal for a Delaware Center for Cognitive Aging Research}

For the past few years, faculty from the University of Delaware and ChristianaCare have conducted collaborative research that span the disciplines of medicine, neuropsychology, physiology, and engineering; with particular emphasis in cognitive and psychosocial 
assessment, ${ }^{35-37}$ compensatory cognitive rehabilitation, ${ }^{27,28,32}$ neurovascular anatomy and physiology, magnetic resonance elastography, ${ }^{38-40}$ and psychiatric interventions for mood and behavior. ${ }^{41-43}$ As discussed in Martens et al. (this issue) there is an effort to formally establish a Delaware Center for Cognitive Aging Research (DECCAR). The mission of this proposed center is to support and advance research, education, and clinical training in Alzheimer's disease and related disorders, to improve the timeliness and accuracy of the diagnosis, therapeutic interventions, person-centered clinical care, and quality of life for patients across the continuum from typical aging to dementia and families. For more information on this proposal, the reader is referred to Martens et al. (this issue) and https://sites.udel.edu/memory-research/. This website also has educational resources for clinicians (e.g., a patient handout on D.A.N.C.E.R.S. protective factors) and ways to refer patients to clinical trials and other research studies in Delaware.

\section{Conclusion}

Alzheimer's disease is and will continue to be a very significant public health problem, currently affecting approximately 19,000 Delawareans. ${ }^{8}$ Although there are no curative or diseasemodifying pharmacological treatments for $\mathrm{AD}$, encouraging findings from the FINGER and MAPT trials have inspired significant efforts in Delaware aimed at behavioral preventive interventions and early identification of atypical cognitive decline. For up-to-date information about these efforts, the interested reader can visit the websites of the Memory Ambassador program (https://christianacare.org/es/swank-memory-ambassadors-program) and the online home of UD memory research, https://sites.udel.edu/memory-research.

\section{References}

1. Salthouse, T. (2012). Consequences of age-related cognitive declines. Annual Review of Psychology, 63, 201-226. PubMed https://doi.org/10.1146/annurev-psych-120710-100328

2. Salthouse, T. A. (2009, April). When does age-related cognitive decline begin? Neurobiology of Aging, 30(4), 507-514. PubMed https://doi.org/10.1016/i.neurobiolaging.2008.09.023

3. Cohen, M. L., Weatherford, S., \& Nandakumar, R. (2019, May 21). How normal are "normal" errors of language and cognition? J Speech Lang Hearing Res, 62(5), 1468-1472. PubMed https://doi.org/10.1044/2018 JSLHR-L-18-0215

4. Begum, A., Dewey, M., Hassiotis, A., Prince, M., Wessely, S., \& Stewart, R. (2014, July). Subjective cognitive complaints across the adult life span: A 14-year analysis of trends and associations using the 1993, 2000 and 2007 English Psychiatric Morbidity Surveys.

Psychological Medicine, 44(9), 1977-1987. PubMed https://doi.org/10.1017/S0033291713002444

5. McAlister, C., \& Schmitter-Edgecombe, M. (2016, September). Cross-sectional and longitudinal analyses of everyday memory lapses in older adults. Neuropsychology, Development, and Cognition. Section B, Aging, Neuropsychology and Cognition, 23(5), 591-608. PubMed https://doi.org/10.1080/13825585.2015.1132669

6. Bott, N. T., Hall, A., Madero, E. N., Glenn, J. M., Fuseya, N., Gills, J. L., \& Gray, M. (2019, September 19). Face-to-face and digital multidomain lifestyle interventions to enhance 
cognitive reserve and reduce risk of Alzheimer's disease and related dementias: A review of completed and prospective studies. Nutrients, 11(9), 2258. PubMed https://doi.org/10.3390/nu11092258

7. Livingston, G., Huntley, J., Sommerlad, A., Ames, D., Ballard, C., Banerjee, S., . . . Mukadam, N. (2020, August 8). Dementia prevention, intervention, and care: 2020 report of the Lancet Commission. Lancet, 396(10248), 413-446. PubMed https://doi.org/10.1016/S0140-6736(20)30367-6

8. Alzheimer's Association. (2020). Alzheimer's disease facts and figures. Alzheimer's \& Dementia, 16(3), 391. https://doi.org/10.1002/alz.12068

9. American Psychiatric Association. (2013). Diagnostic and statistical manual of mental disorders, 5th Ed.

10. Pickett, J., Bird, C., Ballard, C., Banerjee, S., Brayne, C., Cowan, K., .. W Walton, C. (2018, July). A roadmap to advance dementia research in prevention, diagnosis, intervention, and care by 2025. International Journal of Geriatric Psychiatry, 33(7), 900-906. PubMed https://doi.org/10.1002/gps.4868

11. Smith, G. E., \& Bondi, M. W. (2013). Mild cognitive impairment and dementia: definitions, diagnosis, and treatment. Oxford University Press.

12. Kivipelto, M., Solomon, A., Ahtiluoto, S., Ngandu, T., Lehtisalo, J., Antikainen, R., . . . Soininen, H. (2013, November). The Finnish Geriatric Intervention Study to Prevent Cognitive Impairment and Disability (FINGER): Study design and progress. Alzheimers Dement, 9(6), 657-665. PubMed https://doi.org/10.1016/j.jalz.2012.09.012

13. Ngandu, T., Lehtisalo, J., Solomon, A., Levälahti, E., Ahtiluoto, S., Antikainen, R., .. . Kivipelto, M. (2015, June 6). A 2 year multidomain intervention of diet, exercise, cognitive training, and vascular risk monitoring versus control to prevent cognitive decline in at-risk elderly people (FINGER): A randomised controlled trial. Lancet, 385(9984), 2255-2263. PubMed https://doi.org/10.1016/S0140-6736(15)60461-5

14. Kivipelto, M., Mangialasche, F., Snyder, H. M., Allegri, R., Andrieu, S., Arai, H., . . . Carrillo, M. C. (2020, July). World-Wide FINGERS Network: A global approach to risk reduction and prevention of dementia. Alzheimers Dement, 16(7), 1078-1094. PubMed https://doi.org/10.1002/alz.12123

15. van Charante, E. P., Richard, E., Eurelings, L. S., van Dalen, J. W., Ligthart, S. A., van Bussel, E. F., . . van Gool, W. A. (2016, August 20). Effectiveness of a 6-year multidomain vascular care intervention to prevent dementia (preDIVA): A cluster-randomised controlled trial. Lancet, 388(10046), 797-805. PubMed https://doi.org/10.1016/S0140$\underline{6736(16) 30950-3}$

16. Andrieu, S., Guyonnet, S., Coley, N., Cantet, C., Bonnefoy, M., Bordes, S., . .. Vellas, B., $\&$ the MAPT Study Group. (2017, May). Effect of long-term omega 3 polyunsaturated fatty acid supplementation with or without multidomain intervention on cognitive function in elderly adults with memory complaints (MAPT): A randomised, placebo-controlled trial. Lancet Neurol, 16(5), 377-389. PubMed 
17. Livingston, G., Sommerlad, A., Orgeta, V., Costafreda, S. G., Huntley, J., Ames, D., . . . Mukadam, N. (2017, December 16). Dementia prevention, intervention, and care. Lancet, 390(10113), 2673-2734. PubMed https://doi.org/10.1016/S0140-6736(17)31363-6

18. Scheltens, P., De Strooper, B., Kivipelto, M., Holstege, H., Chételat, G., Teunissen, C. E., . . . van der Flier, W. M. (2021, April 24). Alzheimer's disease. Lancet, 397(10284), 15771590. PubMed https://doi.org/10.1016/S0140-6736(20)32205-4

19. Babulal, G. M., Quiroz, Y. T., Albensi, B. C., Arenaza-Urquijo, E., Astell, A. J., Babiloni, C., .... O'Bryant, S. E., \& the International Society to Advance Alzheimer's Research and Treatment, Alzheimer's Association. (2019, February). Perspectives on ethnic and racial disparities in Alzheimer's disease and related dementias: Update and areas of immediate need. Alzheimers Dement, 15(2), 292-312. PubMed

20. Chin, A. L., Negash, S., \& Hamilton, R. (2011, July-September). Diversity and disparity in dementia: The impact of ethnoracial differences in Alzheimer disease. Alzheimer Disease and Associated Disorders, 25(3), 187-195. PubMed https://doi.org/10.1097/WAD.0b013e318211c6c9

21. Yaffe, K., Falvey, C., Harris, T. B., Newman, A., Satterfield, S., Koster, A., . .. Simonsick, E., \& the Health ABC Study. (2013, December 19). Effect of socioeconomic disparities on incidence of dementia among biracial older adults: Prospective study. BMJ (Clinical Research Ed.), 347, f7051-f7051. PubMed

22. Turunen, M., Hokkanen, L., Bäckman, L., Stigsdotter-Neely, A., Hänninen, T., Paajanen, T., ... Ngandu, T. (2019, July 10). Computer-based cognitive training for older adults:

Determinants of adherence. PLoS One, 14(7), e0219541. PubMed https://doi.org/10.1371/journal.pone.0219541

23. Coley, N., Ngandu, T., Lehtisalo, J., Soininen, H., Vellas, B., Richard, E., . .. Andrieu, S., $\&$ the HATICE, FINGER, and MAPT/DSA groups. (2019, June). Adherence to multidomain interventions for dementia prevention: Data from the FINGER and MAPT trials. Alzheimers Dement, 15(6), 729-741. PubMed

24. Heffernan, M., Andrews, G., Fiatarone Singh, M. A., Valenzuela, M., Anstey, K. J., Maeder, A. J., . . .. Brodaty, H., \& the Maintain Your Brain Collaborative Team. (2019). Maintain your brain: Protocol of a 3-year randomized controlled trial of a personalized multi-modal digital health intervention to prevent cognitive decline among community dwelling 55 to 77 year olds. J Alzheimers Dis, 70(s1), S221-S237. PubMed

25. Bahar-Fuchs, A., Martyr, A., Goh, A. M., Sabates, J., \& Clare, L. (2019, March 25). Cognitive training for people with mild to moderate dementia. Cochrane Database Syst Rev, 3, CD013069. PubMed https://doi.org/10.1002/14651858.CD013069.pub2

26. Rebok, G. W., Ball, K., Guey, L. T., Jones, R. N., Kim, H. Y., King, J. W., . ... Willis, S. L., $\&$ the ACTIVE Study Group. (2014, January). Ten-year effects of the advanced cognitive training for independent and vital elderly cognitive training trial on cognition and everyday functioning in older adults. Journal of the American Geriatrics Society, 62(1), 16-24. $\underline{\text { PubMed }}$ 
27. Lanzi, A., Wallace, S. E., Cohen, M. L., \& Bourgeois, M. S. (2021). Structured external memory aid treatment (SEMAT) for older adults with mild cognitive impairment: Longterm adherence and acceptability of treatment. Aphasiology, 1-17.

28. Lanzi, A., \& Bourgeois, M. S. (2020, February 21). Structured external memory aid treatment for mild cognitive impairment. American Journal of Speech-Language Pathology, 29(1S), 474-484. PubMed https://doi.org/10.1044/2019_AJSLP-CAC48-18-0209

29. Greenaway, M. C., Duncan, N. L., \& Smith, G. E. (2013, April). The memory support system for mild cognitive impairment: Randomized trial of a cognitive rehabilitation intervention. International Journal of Geriatric Psychiatry, 28(4), 402-409. PubMed https://doi.org/10.1002/gps.3838

30. Chandler, M. J., Locke, D. E., Crook, J. E., Fields, J. A., Ball, C. T., Phatak, V. S., . . Smith, G. E. (2019, May 3). Comparative effectiveness of behavioral interventions on quality of life for older adults with mild cognitive impairment: A randomized clinical trial. JAMA Network Open, 2(5), e193016. PubMed https://doi.org/10.1001/jamanetworkopen.2019.3016

31. Smith, G. E., Chandler, M., Fields, J. A., Aakre, J., \& Locke, D. E. C. (2018). A survey of patient and partner outcome and treatment preferences in mild cognitive impairment. $J$ Alzheimers Dis, 63(4), 1459-1468. PubMed https://doi.org/10.3233/JAD-171161

32. Lanzi, A., Ellison, J. M., \& Cohen, M. L. (2021). The "counseling+" roles of the speechlanguage pathologist serving older adults with mild cognitive impairment and dementia from Alzheimer's disease. Perspectives of the ASHA Special Interest Groups.

33. Gianattasio, K. Z., Prather, C., Glymour, M. M., Ciarleglio, A., \& Power, M. C. (2019, December 9). Racial disparities and temporal trends in dementia misdiagnosis risk in the United States. Alzheimers Dement (NY), 5(1), 891-898. PubMed https://doi.org/10.1016/j.trci.2019.11.008

34. Lind, K. E., Hildreth, K., Lindrooth, R., Crane, L. A., Morrato, E., \& Perraillon, M. C. (2018, September). Ethnoracial disparities in medicare annual wellness visit utilization: Evidence from a nationally representative database. Medical Care, 56(9), 761-766. PubMed https://doi.org/10.1097/MLR.0000000000000962

35. Cohen, M. L., Lanzi, A. M., \& Boulton, A. J. (2021, June). Clinical use of PROMIS, NeuroQoL, TBI-QoL, and other patient-reported outcome measures for individual clients with cognitive \& language disorders. Seminars in Speech and Language, 42(3), 192-210. PubMed https://doi.org/10.1055/s-0041-1731365

36. Cohen, M. L., Aita, S., Mari, Z., \& Brandt, J. (2015). The unique and combined effects of apathy and depression on cognition in Parkinson's disease. Journal of Parkinson's Disease, 5(2), 351-359. PubMed https://doi.org/10.3233/JPD-140484

37. Cohen, M. L., Tulsky, D. S., Holdnack, J. A., Carlozzi, N. E., Wong, A., Magasi, S., . . Heinemann, A. W. (2017, November). Cognition among community-dwelling individuals with spinal cord injury. Rehabilitation Psychology, 62(4), 425-434. PubMed https://doi.org/10.1037/rep0000140

38. Daugherty, A. M., Schwarb, H. D., McGarry, M. D. J., Johnson, C. L., \& Cohen, N. J. (2020, September). Magnetic resonance elastography of human hippocampal subfields: 
CA3-dentate gyrus viscoelasticity predicts relational memory accuracy. Journal of Cognitive Neuroscience, 32(9), 1704-1713. PubMed https://doi.org/10.1162/jocn_a 01574

39. Hiscox, L. V., Johnson, C. L., McGarry, M. D. J., Marshall, H., Ritchie, C. W., van Beek, E. J. R., .. Starr, J. M. (2020). Mechanical property alterations across the cerebral cortex due to Alzheimer's disease. Brain Communications, 2(1), fcz049. PubMed https://doi.org/10.1093/braincomms/fcz049

40. Delgorio, P. L., Hiscox, L. V., Daugherty, A. M., Sanjana, F., Pohlig, R. T., Ellison, J. M., . . . Johnson, C. L. (2021, May 10). Effect of aging on the viscoelastic properties of hippocampal subfields assessed with high-resolution MR elastography. Cereb Cortex, 31(6), 2799-2811. PubMed https://doi.org/10.1093/cercor/bhaa388

41. Ellison, J. M. (2008). Agitation in dementia: Update and prospectus. The Psychiatric Times, $25(2), 57-57$.

42. Ellison, J. M., Harper, D. G., Berlow, Y., \& Zeranski, L. (2008, January). Beyond the "C" in MCI: Noncognitive symptoms in amnestic and non-amnestic mild cognitive impairment. CNS Spectrums, 13(1), 66-72. PubMed https://doi.org/10.1017/S1092852900016175

43. Ellison, J. M., Kyomen, H. H., \& Harper, D. G. (2012, March). Depression in later life: An overview with treatment recommendations. The Psychiatric Clinics of North America, 35(1), 203-229. PubMed https://doi.org/10.1016/j.psc.2012.01.003

Copyright (c) 2021 Delaware Academy of Medicine / Delaware Public Health Association.

This is an Open Access article distributed under the terms of the Creative Commons Attribution Non-Commercial License (https://creativecommons.org/licenses/by-nc-nd/4.0/) which permits unrestricted non-commercial use, distribution, and reproduction in any medium, provided the original work is properly cited. 